\title{
La presse et le nucléaire, couple infernal
}

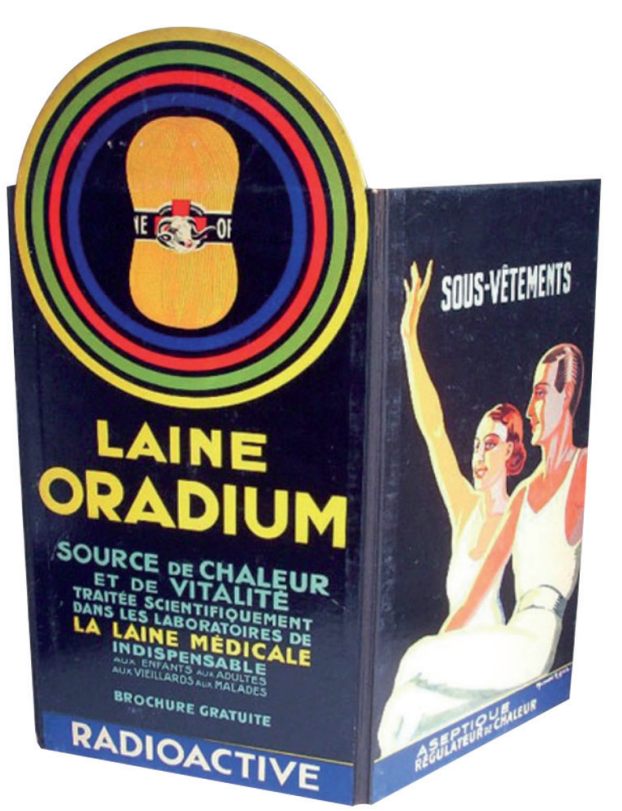

L'électronucléaire incarne jusqu'à la symboliser l'ambivalence de la technologie. Sa puissance lui permet de rendre d'immenses services, sa perte de contrôle peut déboucher sur des dégâts inacceptables. Y recourir ou s'en priver ne pose donc pas seulement la question de la capacité à y accéder, à le maitriser, ou simplement son utilité, mais également celle de son acceptation sociale. En régime politique démocratique, celui souhaité par la population française, elle doit donc également satisfaire à la volonté populaire, exprimée par le suffrage universel lors du choix des législateurs et gouvernants.

Cette exigence semble simple mais se heurte à plusieurs difficultés dont celle de la qualité de l'information des citoyens. Pour que la démocratie ne soit pas un leurre, les choix doivent être opérés « en connaissance de cause ". Ce prérequis démocratique, en ce cas, ne peut se limiter à la forme souvent caricaturale des "professions de foi ", sur une page rectoverso, distribuées peu avant l'élection des représentants du peuple. La presse, censée apporter une contribution décisive au débat démocratique, joue-t-elle son rôle dans celui lié à l'électronucléaire?

Sylvestre Huet, journaliste, auteur du blog $\left\{\right.$ sciences $\left.^{2}\right\}$

L'information des citoyens, tout comme le rôle de contrôle et de contre-pouvoir que la presse incarne, ou devrait incarner, trouve dans le nucléaire une dimension supplémentaire. En effet, la technicité de la matière oblige à un effort d'explication et de médiation scientifique pour aider les citoyens à former leur opinion. Pour autant, l'Histoire a montré que dans le domaine du nucléaire la presse a joué ce rôle avec difficulté, jusqu'à arriver à des cas avérés de désinformation.

\section{Asservissement des populations}

L'histoire de cette interrogation dépasse le demi-siècle, avec des précédents peu encourageants. La découverte et les premières applications de la radioactivité entrainent la publication d'articles vantant les "bienfaits " de cette dernière... dans l'eau de boisson. Au début des années 1950, la «promesse technologique » domine. Répercutant sans esprit critique un discours publicitaire, revues et journalistes annoncent voitures et fusées nucléaires, diffusent l'illusion d'une électricité sans limites et quasi gratuite. Puis, le propos se divise en deux postures opposées. Lors du démarrage du programme nucléaire français de 1974, il est présenté comme la panacée capable de résoudre tous les problèmes économiques du pays... ou à l'inverse comme devant nécessairement entrainer l'asservissement des populations dans une société policière, soumise au secret et détruisant les libertés individuelles et collectives.

\section{Bourdes et désinformations}

Les années récentes ne font guère mieux. L'accident de Fukushima en mars 2011 a donné lieu à de multiples bourdes et désinformations susceptibles d'intéresser les sociologues des médias. Le Figaro annonce en mars 2016 des cancers de la thyroïde chez les enfants, dus à la contamination radioactive, alors qu'il s'agit d'une confusion entre incidence épidémiologique et dépistage systématique ${ }^{(a)}$. Une confusion relevée dans la majorité des articles sur le sujet, malgré l'avertissement clair des spécialistes : "ce n'est que si l'incidence annuelle du cancer de la thyrö̈de chez l'enfant augmente à partir de la période 2016-2018 (ou au cours des périodes suivantes) qu'un lien avec l'accident de Fukushima pourra être évoqué ", explique en effet l'Institut de Radioprotection et de Sureté Nucléaire (IRSN) dans un document destiné aux journalistes et au public.

Le Nouvel Observateur, en aout 2012, sonne le tocsin : "C'est une petite piscine - et un désastre planétaire en puissance. Un cube en béton de onze mètres de profondeur, rempli d'eau et bourré de combustibles 


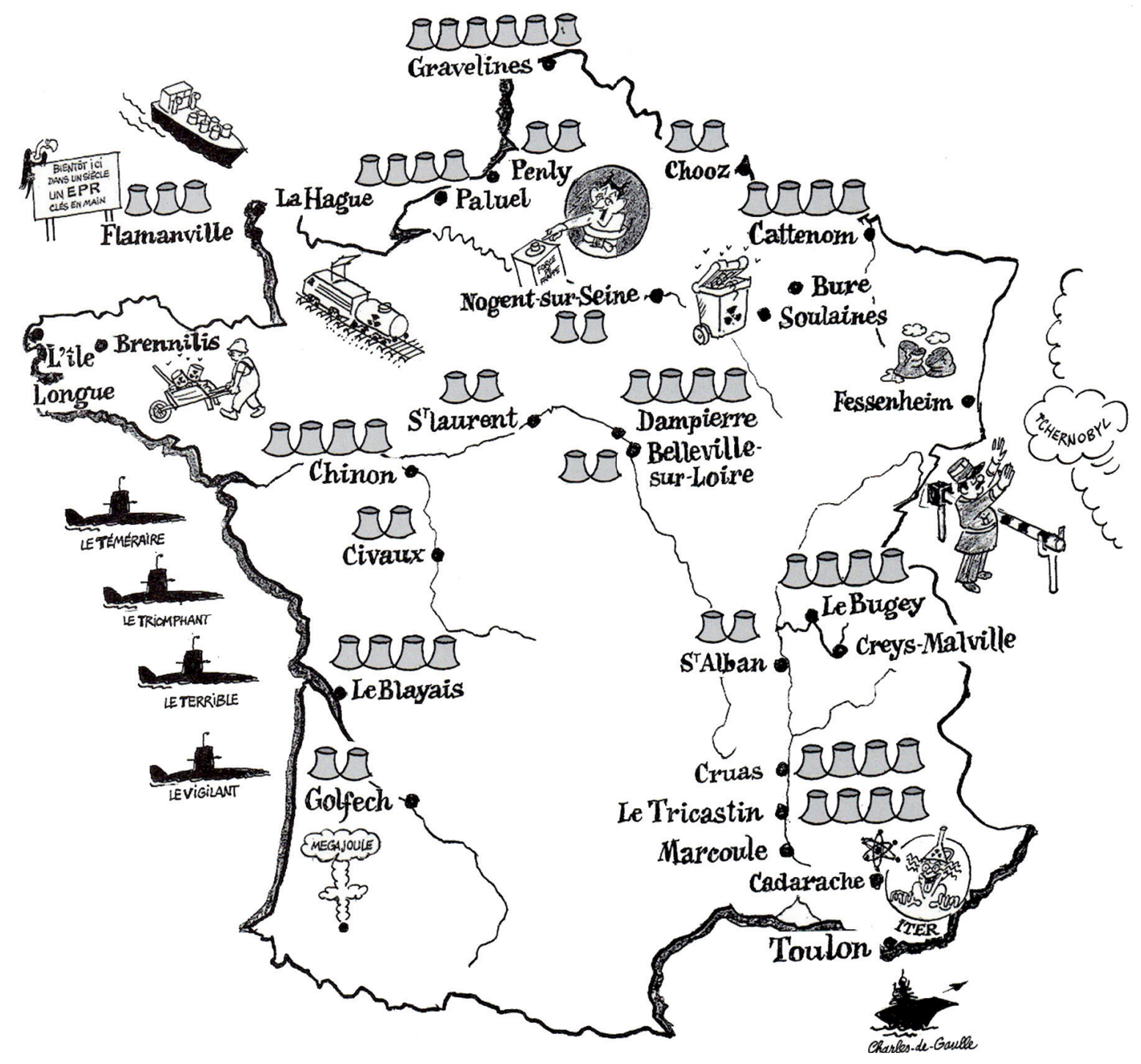

Le nucléaire civil et militaire dans la France de Sarkozy, vu par Cabu. Les réacteurs de recherche, tels que l'Institut Laue-Langevin situé dans la ville de Grenoble, ne sont pas mentionnés.

nucléaires usagés : 264 tonnes de barres très radioactives! Depuis un an et demi, ce bassin dit de "désactivation" repose à trente mètres du sol sur le bâtiment ébranlé du réacteur numéro 4 de la centrale de Fukushima-Daiichi. Il n'est plus protégé ni par un toit solide ni par des murs, mais par une simple bâche de plastique blanche. " Au moment où Le Nouvel Observateur publie cet article alarmiste, la piscine est recouverte d'une structure métallique lourde de 60 tonnes et non d'une bâche en plastique. Elle sera ensuite entièrement vidée de ses combustibles nucléaires. Parmi les errements de la presse à propos de l'accident de Fukushima, l'attribution des 20000 morts du tsunami de mars 2011... à l'accident nucléaire par l'Humanité lors de l'anniversaire de l'évènement, en 2016.
Avec un tel traitement, comment s'étonner de ce que, selon l'enquête sociologique annuelle de l'IRSN ${ }^{(b)}$, une majorité de Français considèrent l'accident japonais comme "plus effrayant " que celui de Tchernobyl, dont le bilan sanitaire est pourtant bien plus grave?

\section{Éléments rationnels}

L'anomalie de fabrication survenue sur le fond et le couvercle de la cuve de l'EPR en construction à Flamanville a provoqué la parution de centaines d'articles. La plupart d'entre eux ont précédé le travail d'analyse des conséquences de cette anomalie sur la capacité de la cuve à remplir sa fonction. Et d'annoncer ou de suggérer que la cuve ne pouvait certainement pas être utilisée... ce qui s'est révélé, in fine, une "information" médiocre. Après l'autorisation donnée par l'Autorité de Sureté Nucléaire (ASN) pour cette cuve, il ne restait plus que l'accusation de collusion avec les industriels contrôlés pour justifier les articles publiés auparavant.

Le débat public sur l'économie ou le bilan des avantages et inconvénients du choix nucléaire pour la France est toujours grevé par une ignorance entretenue. Lorsque la Cour des comptes fait le bilan très complet des couts du nucléaire depuis son origine $^{(\mathrm{c})}$, la presse aligne les milliards... mais sans les mettre en comparaison avec d'autres sources possibles d'électricité. Les anciens " prélèvements sur les bénéfices " puis les milliards de 
$\gg\rangle$

dividendes versés à l'État depuis 2006 par EDF, dont l'origine se situe pour l'essentiel dans la production nucléaire, sont ignorés. Un calcul simple, comme celui consistant à comparer le cout du " grand carénage » et des mesures postFukushima à celui d'un investissement identique en capacités de production assurant la continuité de l'approvisionnement n'est jamais fait (voir encadré, $N d E$ ).

Du coup, Le Monde en vient à titrer sur une mystérieuse "Obsession française " pour expliquer le choix d'un socle nucléaire majoritaire pour l'électricité, opéré depuis 1974 par tous les gouvernements et majorités parlementaires. Voire à évoquer le nucléaire militaire comme source de cette obsession. Pourtant, l'économie, le cout de l'électricité pour les entreprises et les ménages comme la volonté initiale de desserrer l'étau de la contrainte extérieure - financière et d'approvisionnement alors en pétrole suffisent à expliquer ce choix par des éléments discutables mais rationnels.

À ces éléments s'ajoute, depuis que la communauté internationale a pris conscience du problème climatique l'avantage crucial d'une source d'électricité décarbonée. Mais l'enquête sociologique annuelle de l'IRSN indique que près de la moitié des Français persistent à croire que les centrales nucléaires contribuent "beaucoup ou assez " à ce changement climatique. Si la presse n'est pas la seule responsable de cette incompréhension massive, comment l'exonérer totalement de cet état piteux de la diffusion des connaissances de base sur ce sujet crucial ?

\section{Conversation citoyenne}

Pourquoi la presse fait-elle si mal son travail sur ce sujet $^{(d)}$ ? Les raisons en sont multiples, des choix idéologiques à l'incompétence, en passant par la difficulté objective du sujet qui exige un investissement en temps rarement disponible pour les journalistes. Elles sont accentuées par le total désintérêt pour la technologie, et souvent même pour l'appareil productif, de la plupart des rédactions en chef. Estelle pour autant la seule responsable de l'état du débat démocratique sur le sujet? Ce serait là une conclusion beaucoup trop indulgente pour les industriels. Ces derniers ont en effet usé et abusé du discours publicitaire cachant les difficultés réelles, comme cette présentation récurrente des déchets nucléaires réduits au volume "d'une piscine olympique ». Au regard des dimensions du projet CIGEO d'enfouissement de ces déchets - des galeries souterraines de plusieurs dizaines de kilomètres, des alvéoles de plusieurs centaines, des installations de surface de taille industrielle - la volonté de duper est patente. Si la loi et les règles obligent les industriels à déclarer chaque incident aux autorités, le discours tenu vise systématiquement à minimiser les risques. À chaque incident ou problème technique rencontré les industriels privilégient des " éléments de langage " typiques d'une démarche publicitaire ou propagandiste au détriment d'une information détaillée et honnête. D'expérience personnelle, nombre de dirigeants d'EDF estiment les Français (voire les journalistes) trop "bêtes" pour comprendre la technologie qu'ils utilisent. D'où le recours aux ficelles publicitaires plutôt qu'à l'exposé rationnel. Si les industriels apparaissent toujours réagir à des informations diffusées par l'ASN et l'IRSN, c'est qu'ils ne prennent jamais l'initiative de faire part des difficultés rencontrées et encore moins de leurs erreurs ou fautes, comme on a pu le constater dans l'affaire des falsifications de documents relatifs à la fabrication de composants lourds à l'usine de Creusot Forge, avant et après son acquisition par Areva.

Cette attitude s'oppose à celle de l'ASN et de l'IRSN qui constituent à l'inverse de précieuses sources d'informations fiables pour les journalistes. Lors de l'accident de Fukushima, les propos lénifiants des directions d'Areva et d'EDF tranchaient abruptement avec ceux de l'ASN, réalistes sur la dimension de la catastrophe. Soulignons toutefois un paradoxe : il n'est pas interdit de voir dans la sévérité et la capacité à " parler vrai » de l'ASN (autorité administrative indépendante depuis 2006) et de l'IRSN un effet positif de l'exigence de sureté très forte de la population française dont témoigne, avec ses défauts, la couverture médiatique du risque nucléaire.

a. Le dépistage systématique montre une incidence de 11 cancers de la thyroïde pour 100000 enfants et par an dans la préfecture du Fukushima contre 23 à 130 dans trois autres préfectures (Aomori, Hiroshima et Yamanashi) indemnes de contamination pour la période 2011/2014

b. http://barometre.irsn.fr/barometre2017/\#page=1 c. Voir dans ce dossier l'article d'A.-S. Dessillons (p. 29). d. NdE : voir en fin de dossier (p. 62) quelques exemples de travaux de presse, y compris critiques du nucléaire, réalisés de façon compétente.

e. Voir dans ce dossier l'article de J. Percebois (p. 52) f. Voir dans ce dossier l'article de N. Maïzi et F. Briens (p. 49).

\section{Éléments pour le calcul proposé par Sylvestre Huet}

La Cour des comptes estime à 100 milliards d'euros le « grand carénage » (75 en investissement + 25 en exploitation) d'ici 2025 , soit approximativement 1,7 milliards d'euros par réacteur. Or d'ici 2025, 34 réacteurs vont atteindre la limite des 40 ans, ce qui représente $31,6 \mathrm{GW}$ installés, à remplacer s'ils ne sont pas rénovés. II faudrait pour cela construire environ 20 EPR de 1,6 GW. En supposant qu'en production régulière le cout d'un EPR tombe à 5,6 milliards d'euros (soit 3500 euros/kW installé), le cout total serait de l'ordre de 112 milliards d'euros.

Les deux couts estimés, bien qu'ils ne constituent qu'un ordre de grandeur approximatif, sont donc comparables. Mais construire, dans ces sept années, une vingtaine d'EPR apparait comme une mission impossible. Si on construit plutôt de l'éolien ou du photovoltaïque, il faut inclure dans le calcul les couts des installations supplémentaires au gaz naturel pour compenser l'intermittence, et le redimensionnement du réseau pour tenir compte des pics de production à absorber pour ne pas perdre l'électricité produite ${ }^{(\mathrm{e})}$. L'autre solution étant de diminuer la consommation d'électricité(f).

Remerciements à Roland Lehoucq, NdE. 